\title{
Dosimetric Study on Variations of Gamma Index (GI) in Pre-Treatment Verification Procedure in Intensity Modulated Radiotherapy (IMRT) Plans with Varying Grid Sizes Using 2D Array Detectors
}

\section{Pankaj Pathak $^{1 *}$, Praveen Kumar Mishra ${ }^{2}$ and Narendra Birbiya ${ }^{3}$}

${ }^{1}$ Birla Institute Of Medical Research Hospital, Gwalior, Madhya Pradesh, India

${ }^{2}$ Department of Raditherapy, G.R . Medical College, Gwalior Madhya Pradesh, India

${ }^{3}$ Department of Radiation Oncology, Birla Institute Of Medical Research, Surya Mandir Road, Gwalior Madhya Pradesh, India

\section{Abstract}

Aim: The main aim of our study was to compare the variation of Gamma Index (GI) in Pre-treatment Verification Procedure in Intensity Modulated Radiotherapy (IMRT) Plans with Varying Grid Sizes Using 2D Array Detectors.

Choosing an optimum grid size plays a vital role for planning in radiotherapy cases especially while treating with IMRT. A minimal change of even $1 \mathrm{~mm}$ of grid size can result in large variation in treatment planning and is reflected in quality assurance results also.

Methods and Material: We compared IMRT plans for a total of 12 patients. Out of these 12 patients 4 were head and neck, 4 were pelvic and 4 were brain patients respectively. For each patient three plans were generated with three different grid sizes. The plan acceptance criteria were $95 \%$ of PTV to receive at least $95 \%$ of prescribed dose and dose to $1 \%$ of PTV not to exceed $107 \%$ of prescribed dose. Dose for the organs at risk were respected as per the QUANTEC guidelines. After plan acceptance corresponding Pre-treatment Verification Procedure for IMRT was executed by PTW 729 array detector. The Gamma index (GI) results of each plan were recorded for the three different grid sizes. The passing criteria were kept to be $3 \%$ Dose Difference (DD) and $3 \mathrm{~mm}$ Distance to Agreement (DTA) for all cases.

Results: We estimated the variations in GI quality assurance results for patients undergoing IMRT planning with varying grid sizes of $3 \mathrm{~mm}, 5 \mathrm{~mm}$ and $10 \mathrm{~mm}$ respectively. We also evaluated $2 \%$ DD and $2 \mathrm{~mm} \mathrm{DTA,} 3 \% \mathrm{DD}$, $3 \mathrm{~mm}$ DTA and $5 \%$ DD, $5 \mathrm{~mm}$ DTA criteria for passing result.

Stastical analysis: We have calculated the average and standard Deviations (Std. Dev.) for each passing criteria for $2 \%$ DD, $2 \mathrm{~mm}$ DTA, 3\% DD, $3 \mathrm{~mm}$ DTA and 5\% DD, $5 \mathrm{~mm}$ DTA for each IMRT plans with varying grid sizes.

Conclusions: Though the present results suggest the gamma criteria of $5 \%$ DD and $5 \mathrm{~mm}$ DTA as the most suitable criteria for IMRT quality assurance. This gamma criterion of $5 \%$ DD and $5 \mathrm{~mm}$ DTA favourably exceeds $95 \%$ in each case and grid sizes but it is not recommended for strict verification procedure in Intensity Modulated Radiotherapy (IMRT). The criteria of $2 \%$ DD and $2 \mathrm{~mm}$ DTA, and $3 \%$ DD and $3 \mathrm{~mm}$ DTA gamma values show below $90 \%$ for $5 \mathrm{~mm}$ and $10 \mathrm{~mm}$ grid sizes but exceeds $95 \%$ for the $3 \mathrm{~mm}$ grid sizes.

Hence $3 \mathrm{~mm}$ or less grid sizes should be routinely used in pre-treatment verification procedure IMRT plans using 2D Array Detectors.

Keywords: Gamma index; Dose grid; 2D Array; Radiotherapy; Patient specific quality assurance

\section{Introduction}

The advantages of intensity-modulated radiation therapy (IMRT) in the treatment of head-and-neck cancer (HNC) have been showed in a number of studies [1-3]. High dose to high-risk volumes can be achieved with highly conformal radiation, while sparing adjacent organs at risk (OAR) such as the parotid glands. Clinically it has been shown that IMRT reduces grade- 3 xerostomia compared to threedimensional conformal radiotherapy (3D CRT) $[4,5]$. That is the reason; IMRT has become the standard treatment in many centers. The differences among planned and delivered dose distributions have to be minimized since IMRT dose distributions, with steep dose gradients, are very sensitive to geometrical uncertainties.

The complexity of intensity-modulated radiotherapy (IMRT) demands thorough verification of planned radiation dose before treatment. The film dosimetry for pretreatment verification of patient- specific IMRT dose distribution is gradually being replaced by twodimensional (2D) detector arrays due to their ease of use and immediate readout of the results. For pretreatment verification of IMRT plans 2D detector arrays consisting of a large number of ionization chambers or diodes have been reported. To compare two-dimensional dose distribution, the concept of distance to agreement (DTA), applied in a software tools. Portal Dosimetry (PD) was performed from 12 IMRT

*Corresponding author: Pankaj Pathak, Chief medical Physicist, BIMR Hospitals Radiation Oncology, Surya mandir road, Gwalior, Madhya Pradesh 474004, India, Tel: 07512405715/09753109147; E-mail: pankaj2002@hotmail.com

Received March 25, 2015; Accepted May 23, 2015; Published May 25, 2015

Citation: Pathak P, Mishra PK, Birbiya N (2015) Dosimetric Study on Variations of Gamma Index (GI) in Pre-Treatment Verification Procedure in Intensity Modulated Radiotherapy (IMRT) Plans with Varying Grid Sizes Using 2D Array Detectors. J Cancer Sci Ther 7: 161-166. doi:10.4172/1948-5956.1000342

Copyright: $\odot 2015$ Pathak P, et al. This is an open-access article distributed unde the terms of the Creative Commons Attribution License, which permits unrestricted use, distribution, and reproduction in any medium, provided the original author and source are credited. 
Citation: Pathak P, Mishra PK, Birbiya N (2015) Dosimetric Study on Variations of Gamma Index (GI) in Pre-Treatment Verification Procedure in Intensity Modulated Radiotherapy (IMRT) Plans with Varying Grid Sizes Using 2D Array Detectors. J Cancer Sci Ther 7: 161-166. doi:10.4172/1948-5956.1000342

plans of various clinical sites at gantry zero and Source-to-axis Distance (SAD) of $100 \mathrm{~cm}$

By measuring the closest distance between one dose distributions to where the second distribution has the same dose level the DTA is typically computed. The DTA which is a non-local function, meaning that when it is evaluated at a point, the other distribution is queried at a distance. While the Dose Difference (DD) merely changes sign when the two dose distributions are swapped, the DTA is not the same value of the roles of the two distributions are swapped. It is therefore useful to label the two distributions as the standard and evaluated distributions. The standard distribution is the one whereby the DTA is computed point by point, while the evaluated distribution is the one queried for the closest approach of the specific isodose.

Due to the impact of steep dose gradients, the DDs may not be clinically relevant, but the meaning behind the numbers is clear. The DTA is highly sensitive in shallow dose gradient regions whereas the DD highly is sensitive in steep dose gradient regions. As an example, if the reference dose point has dose $\mathrm{D}$, lying in a shallow dose gradient region, and the evaluated dose at that point is $D+\Delta D$, the distance in the evaluated distribution from the reference point that has the same dose $\mathrm{D}$ may be very far away.

The DTA will therefore be very large, even if $\Delta \mathrm{D}$ is small and has no clinical consequence. Because of the large values in shallow dose gradient regions, regions that typically dominate IMRT dose distributions; the DTA distribution is difficult, if not impossible to interpret by eye.

The DTA can be interpreted as the distance between the two dose distributions in steep dose gradient regions. This interpretation is based on the assumption that the distance is caused primarily by a spatial offset between the two distributions. For distributions that differ by such an offset, the DTA distribution provides an effective and accurate measurement of the offset. Measurements of typical IMRT beams that are modulated by compensators and MLCs are presented with comparisons to treatment planning system dose calculations. A time analysis is done for typical IMRT quality assurance measurements. The setup, calibration, and analysis time for the 2-D diode array are on the order of $20 \mathrm{~min}$, depending on numbers of fields. This is significantly less time than required to do similar analysis with radiographic film. The 2-D diode array is ideal for per-plan quality assurance after an IMRT system is fully commissioned [6].

One of the nice features of the dose difference distribution is its relatively straightforward interpretation. Most physicists can glance at a dose difference distribution and see and interpret the data. The present study will highlight the importance in choosing the grid sizes in pre-treatment verification as well as in IMRT planning. At the same time the planner will have the knowledge of variation in the Gamma Index (GI) with varying grid sizes.

\section{Materials and Methods}

\section{CT acquisition and contouring}

CT scans were acquired using a Somatom Power Spirit CT Simulator (Siemens) with 3-5 mm slice spacing. Patients were in the supine position and immobilized with a thermoplastic head-shoulder mask. A planning CT scan (CT) was acquired one week before RT treatment. The Oncentra version 4.3 (Nucletron) treatment planning system was used for delineation and dose distribution calculations. Target volumes and normal tissues were manually contoured by a physician on each axial slice of the CT using MRI or contrast-enhanced
CT. The definition of volumes was in accordance with ICRU Reports 50-62, but dose-volume parameters were reported according to the new ICRU Report 83 IMRT recommendations. Gross Tumour Volume (GTV) included the primary tumour and affected lymph nodes. The GTV was expanded to include the high-risk regions (CTV).

To compensate for geometrical uncertainties such as setup and organ motion, a $5 \mathrm{~mm}$ margin was automatically added to CTV to obtain the Planning Target Volume (PTV). In order to avoid dose compensation in the build-up region, in cases with no skin infiltration, the PTVs were manually modified excluding areas where the distance to the skin was less than $3 \mathrm{~mm}$. Although these modified PTV were used during optimization process, the absorbed dose was reported over the whole PTV. Prescribed doses were 6,600cGy, 5,000cGy and 5,400cGy for head-and -neck, pelvis (Carcinoma Cervix), and brain respectively.

The critical structures contoured were: the parotid glands, spinal cord, mandible, eyes, oral cavity, brainstem, brain, optic nerves, optic chiasm, bladder, rectum, bowel and femur heads.

\section{Treatment planning}

IMRT treatment plans were generated on the CT with nine $6 \mathrm{MV}$ fields on the Oncentra Treatment Planning System (TPS). For each of the calculation grid sizes, three different sites; namely, head -andneck, cervix, and brain were analyzed. The IMRT plans were optimized using an inverse planning algorithm. The final dose distribution was calculated using the Pencil Beam (PB) with heterogeneity correction and 3-10 $\mathrm{mm}$ grid resolution. Comparison was done first by using $3 \mathrm{~mm}$ calculation grid as a golden standard and keeping the same number of Monitor Units (MUs) per beam for each grid size, then the second part involved renormalizing plans to have the same target coverage (95\% of the prescription dose covering at least $95 \%$ of the target volume) for each grid size used.

Future study plans include their verification with the PTW 2D Array. Pre-treatment dosimetric verification was carried out on fieldper-field and total IMRT plan basis measuring 2D dose distributions in RW3 solid water phantom (PTW-Freiburg, Germany). For 12 patient plans, the dose distribution was re-calculated with the phantom CT scan and delivered to the phantom with the original and 0 degrees gantry, collimator and table angles. Thus IMRT quality control (QC) plans were generated. Dose distribution measurements were measured with 2D-Array seven29 (PTW-Freiburg, Germany) two dimensional ionization chamber systems. Verification measurements of total IMRT plans and each individual beams were compared with expected dose maps, and differences were evaluated by Verisoft program (PTWFreiburg, Germany). To provide comparisons of multidimensional dose distributions, dose comparison tools such as gamma dose distribution, distance- to- agreement (DTA) and DD (DD) have been developed. The gamma dose distribution tool was used in our study. Three different gamma criteria of DD (DD) and distance to agreement (DTA) $(2 \%, 2 \mathrm{~mm}, 3 \% / 3 \mathrm{~mm}$ and $5 \% / 5 \mathrm{~mm} \mathrm{DD} / \mathrm{DTA})$ are selected and graph has been plotted between passing criteria (\%) vs. $2 \% / 2 \mathrm{~mm}, 3 \% / 3$ $\mathrm{mm}$ and $5 \% / 5 \mathrm{~mm}$ DD/DTA as shown in Graph I(a),(b),(c), II (a),(b),(c) and III (a),(b),(c). Criterion validity accepted as section with gamma value less than or equal to $1(\gamma=1)$ to be $90 \%$. IMRT quality control plans of treatment plans which were prepared with Oncentra software, were measured with Elekta Synergy linear accelerator (LINAC) at the same conditions with CT image, by using PTW 2D-Array seven29. Isocentre was located on the top of ionization chambers in array. The measurements were performed in relative mode. Each field has been 


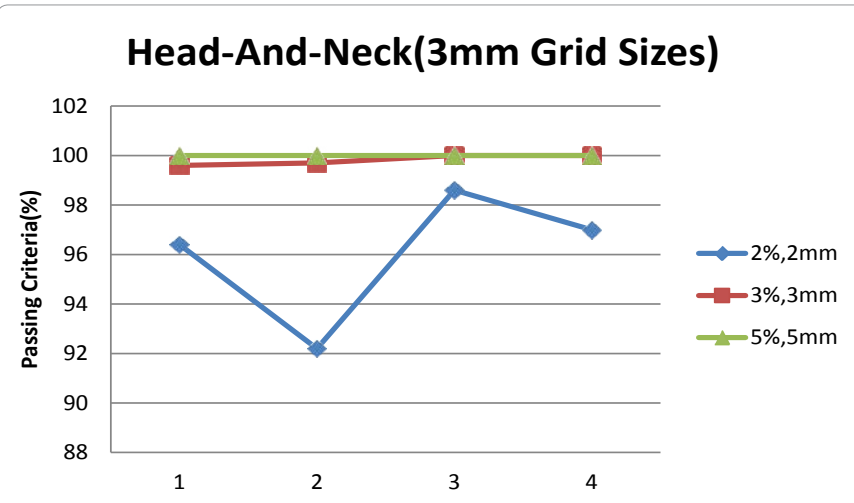

(a)

\section{Head-And-Neck(5mm Grid Sizes)}

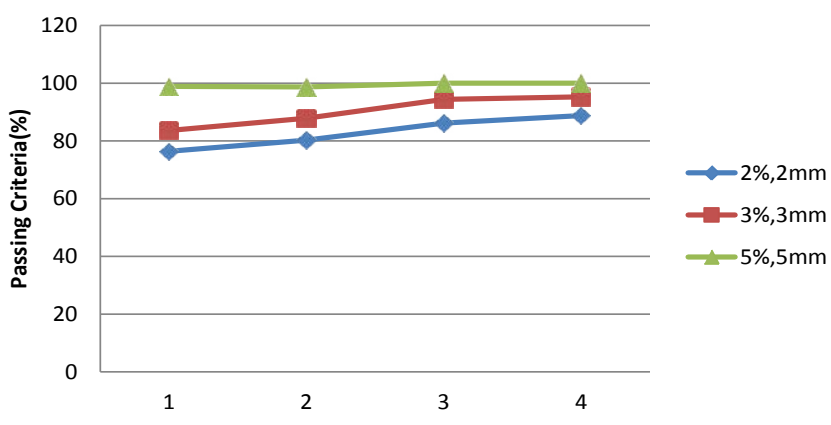

(b)

Head-And-Neck(10mm Grid sizes)

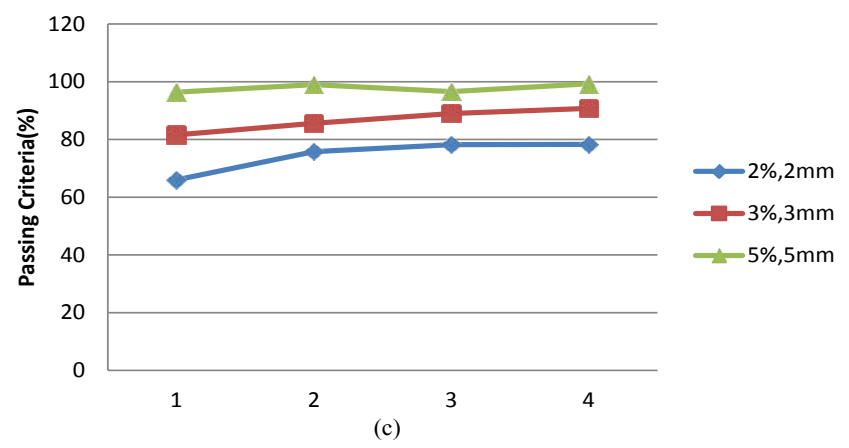

Graph 1: (a), (b), (c) showing the passing criteria (\%) of Head-and-Neck with $3 \mathrm{~mm}, 5 \mathrm{~mm}$ and $10 \mathrm{~mm}$. Grid sizes with $2 \%, 2 \mathrm{~mm}, 3 \%, 3 \mathrm{~mm}$ and $5 \%, 5 \mathrm{~mm}$ dose difference and DTA.

verified in solid water phantoms, with beam incidence perpendicular to the phantom surface. Planar dose distributions of total IMRT plans and each treatment fields, at $5 \mathrm{~cm}$ depth and with $95 \mathrm{~cm}$ SSD in a solid water phantom with gantry, collimator and table angles set to $0^{\circ}$ were obtained with PTW 2D-Array and compared with TPS calculated dose distributions under identical geometric setup. Measurement assembly consisting of PTW 2D-Array seven29 and PTW RW3 solid water phantom is shown in (Figure 1) [7].

2D-array: PTW 2D-array 729 consisting of a plan matrix of $27 \times$ 27 air-filled ionization chambers is used (PTW, Freiburg, Germany). The detector spacing (center to center) is $1 \mathrm{~cm}$. The dimensions of each detector are $0.5 \times 0.5 \times 0.5 \mathrm{~cm}^{3}[8]$.

Verisoft software: The Verisoft software assists physicist $s$ in comparing dose distributions in IMRT verification phantom with dose distributions computed by radiotherapy treatment planning system. Matrices of measured and calculated points of an IMRT beam are compared by subtracting the matrices and visualizing the results. The software supports the gamma evaluation method, it helps in locating hot and cold spots and determines maximum and average deviation between a calculated and measured plan.

A "reasonable" choice of a specific combination of gamma evaluation and acceptance criteria should be based on the accuracy of the applied measurement procedure, its workload, and the ability to detect problem areas in the intended dose distribution. Combinations of gamma evaluation and acceptance criteria depend on many factors including the dosimetric equipment, calculation and measurement
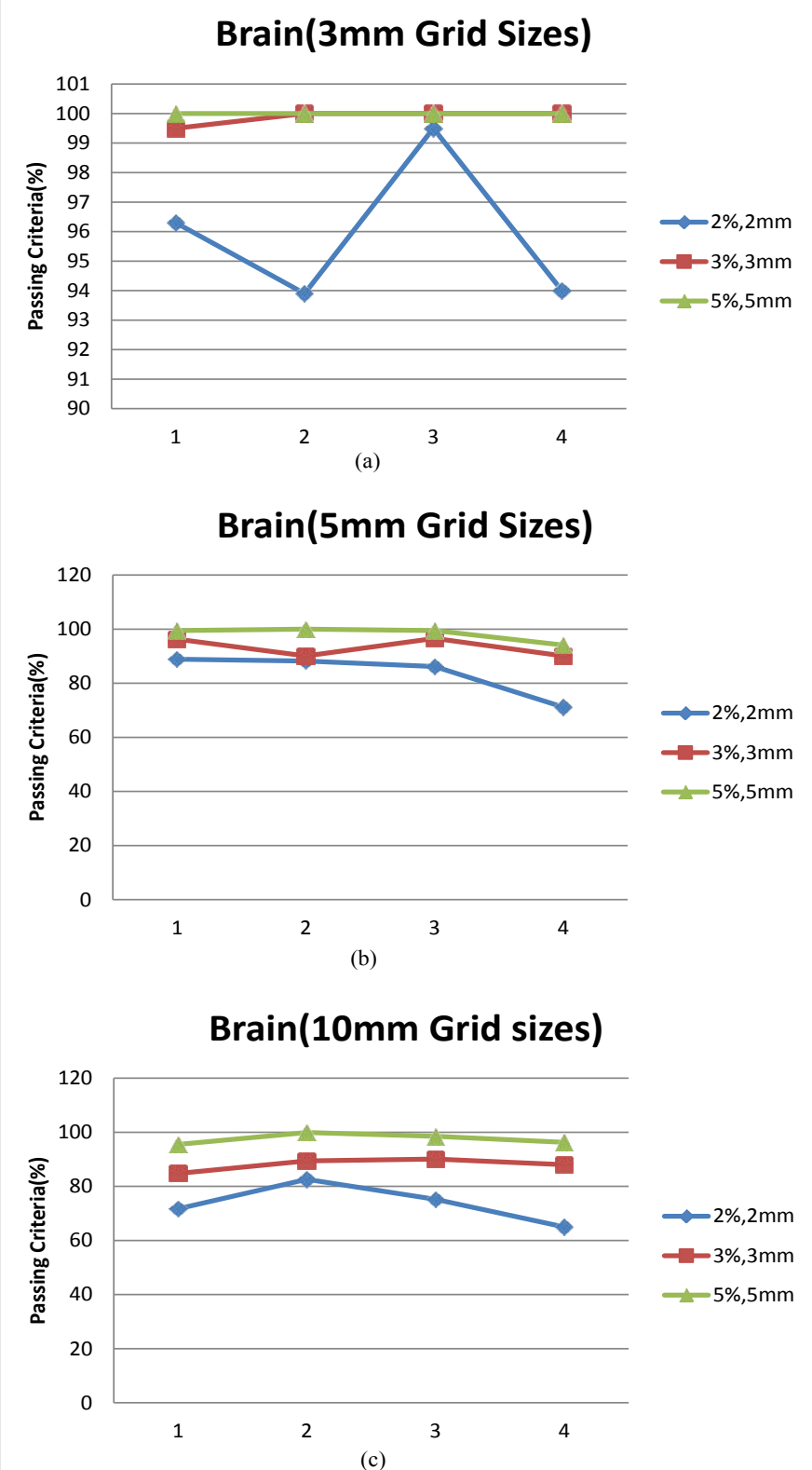

Graph 2: (a), (b), (c) showing the passing criteria (\%) of Brain with $3 \mathrm{~mm}, 5$ $\mathrm{mm}$ and $10 \mathrm{~mm}$. Grid sizes with $2 \%, 2 \mathrm{~mm}, 3 \%, 3 \mathrm{~mm}$ and $5 \%, 5 \mathrm{~mm}$ dose difference and DTA. 
Citation: Pathak P, Mishra PK, Birbiya N (2015) Dosimetric Study on Variations of Gamma Index (GI) in Pre-Treatment Verification Procedure in Intensity Modulated Radiotherapy (IMRT) Plans with Varying Grid Sizes Using 2D Array Detectors. J Cancer Sci Ther 7: 161-166. doi:10.4172/1948-5956.1000342

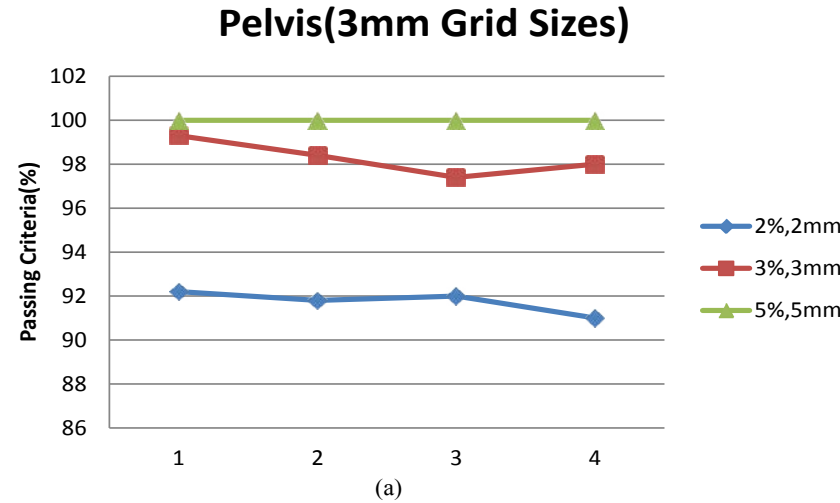

Pelvis(5mm Grid Sizes)

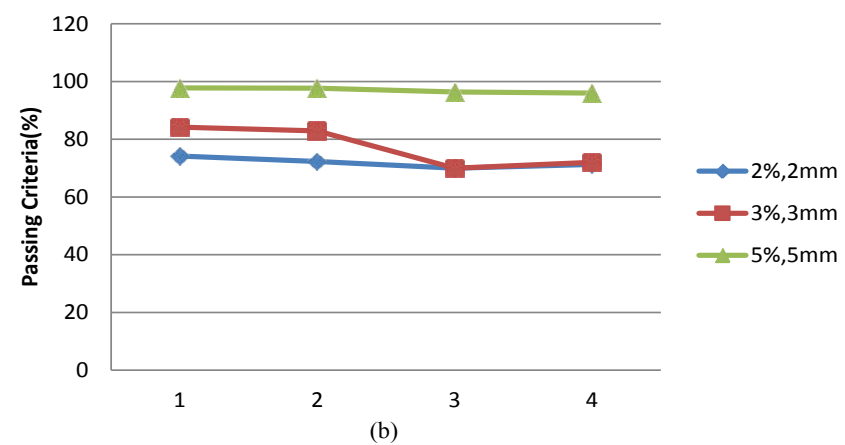

Pelvis(10mm Grid Sizes)

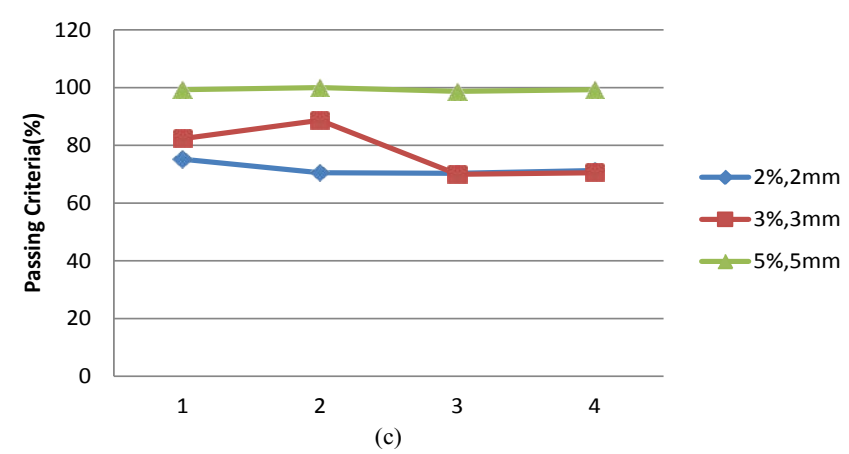

Graph 3: (a), (b), (c) showing the passing criteria (\%) of Pelvis(Ca.Cx) with 3 $\mathrm{mm}, 5 \mathrm{~mm}$ and $10 \mathrm{~mm}$ Grid sizes with $2 \%, 2 \mathrm{~mm}, 3 \%, 3 \mathrm{~mm}$ and $5 \%, 5 \mathrm{~mm}$ dose difference and DTA.

grid, and the data analysis software. It is therefore virtually impossible to provide general recommendations applicable for all situations. In this study, the Verisoft verification software is used to compare gamma distribution for calculated dose (cGy) using TPS and measured dose using films and 2D-array. This is to find out what percent of pixels passing certain criteria imitate a good quality plan. Two tests are performed for the comparison between measured dose from Linac and calculated dose from TPS. The IMRT delivery quality assurance mostly consists of delivering the IMRT plan to a phantom and then comparing the $2 \mathrm{D}$ dose distribution calculated by the TPS with the dose measured using 2D-array or films [9-14].

Optimization goals were as follows: 1) prescription doses (Dpres) must encompass at least $95 \%$ of target volumes; 2) near-minimum absorbed doses (D98\%) of PTVs should be higher than 92\% of Dpres; 3 ) the near-maximum absorbed dose (D2\%) of the PTVs should be less than $110 \%$ of Dpres.

High priority constraints to normal critical structures were: No more than $1.0 \mathrm{~cm}^{3}$ of spinal cord could receive more than $46 \mathrm{~Gy}$;2) no more than $1 \%$ of brainstem could receive more $54 \mathrm{~Gy}$; 3 ) the parotid gland volume receiving $26 \mathrm{~Gy}$ should be less than $50 \%$ in at least one gland; 4) optic nerves Dmax should be less than 56Gy 5) optic chiasm Dmax should be less than 54Gy 6) Bowel $195 \mathrm{cc}$ should be less than $45 \mathrm{~Gy}$; 7) bladder Dmax should be less than 45Gy; 8) rectum Dmax should be less than $50 \mathrm{~Gy}$; 4) D2\% of normal tissue should be less than Dpres.

Low priority constraints that should not compromise target coverage were: 1 ) eyes Dmax should be less than 50 Gy;

\section{Results}

Table 1 (a) (b) and (c) summarises the passing criteria in percentages (\%) for $3 \mathrm{~mm}, 5 \mathrm{~mm}$ and $10 \mathrm{~mm}$ grid size for HeadAnd- Neck ,Brain and Pelvis (Ca.Cx.) for IMRT plans of 12 patients respectively for $2 \% \mathrm{DD}$ and $2 \mathrm{~mm}$ DTA, $3 \% \mathrm{DD}$ and $3 \mathrm{~mm}$ DTA and $5 \% \mathrm{DD}$ and $5 \mathrm{~mm}$ DTA.

The average passing result for all cases were $94.575 \%, 99.325 \%$, and $100 \%$ for $(2 \mathrm{~mm}$ DTA and $2 \%$ of DD, 3\% DD, $3 \mathrm{~mm}$ DTA and 5\% DD, 5 mm DTA) for $3 \mathrm{~mm}$ grid sizes, $79.50 \%, 86.95 \%$ and $98.216 \%$ for $(2 \mathrm{~mm}$ DTA and $2 \%$ of DD, $3 \%$ DD, $3 \mathrm{~mm}$ DTA and $5 \%$ DD, $5 \mathrm{~mm}$ DTA) for $5 \mathrm{~mm}$ grid sizes and $73.34 \%, 84.15 \%$ and $98.23 \%$ (2 $\mathrm{mm}$ DTA and $2 \%$ of $\mathrm{DD}, 3 \% \mathrm{DD}, 3 \mathrm{~mm}$ DTA and 5\% DD,5 mm DTA) for 10mm grid sizes respectively. Hence we conclude that $3 \mathrm{~mm}$ grid size plans has the best passing result with $2 \mathrm{~mm}$ DTA and $2 \%$ of DD, $3 \% \mathrm{DD}, 3 \mathrm{~mm} \mathrm{DTA}$ and $5 \% \mathrm{DD}, 5 \mathrm{~mm}$ DTA when compared with that of $5 \mathrm{~mm}$ and $10 \mathrm{~mm}$ grid sizes (Table 2 ).

\section{Conclusions}

The two dose distributions are the reference and evaluated distributions. The reference distribution is intended to be the one relative to which the other is compared. It is typically the measurement in a measurement versus calculation, or it can be a Monte Carlo calculation when calculations are compared against each other. The reference distribution is queried point by point, and GI computed individually for each. Therefore, the reference distribution can be a single point, for example an ionization chamber measurement point. The evaluated distribution needs to be a distribution, and in early GI papers, it was assumed that it had to have a relatively high spatial resolution. This is because the GI calculation can have relatively large errors in steep dose gradient regions when the spacing between evaluated distribution points is close to the DTA criterion.

Though the present results suggest the gamma criteria of $5 \%$

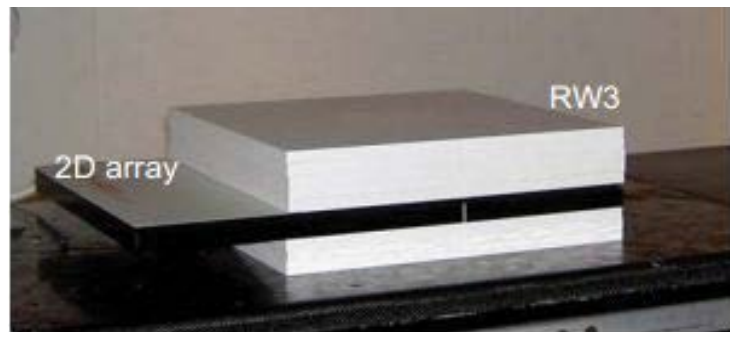

Figure 1: PTW 729 RA Detector Phantom. 
Citation: Pathak P, Mishra PK, Birbiya N (2015) Dosimetric Study on Variations of Gamma Index (GI) in Pre-Treatment Verification Procedure in Intensity Modulated Radiotherapy (IMRT) Plans with Varying Grid Sizes Using 2D Array Detectors. J Cancer Sci Ther 7: 161-166. doi:10.4172/1948-5956.1000342

\begin{tabular}{|c|c|c|c|c|c|c|c|c|c|}
\hline & & & & & \multicolumn{5}{|c|}{ HEAD-AND-NECK } \\
\hline & & & & & & \multicolumn{4}{|c|}{ GRID SIZES(mm) } \\
\hline \multirow[t]{7}{*}{ (a) } & \multirow[b]{2}{*}{$\begin{array}{c}\text { Gamma Function Test } \\
\text { Result (\%) }\end{array}$} & \multicolumn{2}{|c|}{3} & \multirow[b]{3}{*}{$2 \%, 2 \mathrm{~mm}$} & \multirow[t]{2}{*}{5} & \multirow[b]{3}{*}{$5 \%, 5 \mathrm{~mm}$} & \multicolumn{3}{|c|}{10} \\
\hline & & \multirow[b]{2}{*}{$3 \%, 3 \mathrm{~mm}$} & \multirow[b]{2}{*}{$5 \%, 5 \mathrm{~mm}$} & & & & & \multirow[b]{2}{*}{$3 \%, 3 \mathrm{~mm}$} & \multirow[b]{2}{*}{$5 \%, 5 \mathrm{~mm}$} \\
\hline & $2 \%, 2 \mathrm{~mm}$ & & & & $3 \%, 3 \mathrm{~mm}$ & & $2 \%, 2 \mathrm{~mm}$ & & \\
\hline & 96.4 & 99.6 & 100 & 76.4 & 83.6 & 98.9 & 66 & 81.6 & 96.4 \\
\hline & 92.2 & 99.7 & 100 & 80.3 & 87.9 & 98.7 & 75.8 & 85.6 & 99 \\
\hline & 98.6 & 100 & 100 & 86.2 & 94.4 & 100 & 78.2 & 89 & 96.6 \\
\hline & 97 & 100 & 100 & 88.8 & 95.3 & 100 & 78.3 & 90.8 & 99.3 \\
\hline Average & 96.05 & 99.825 & 100 & 82.95 & 90.3 & 99.4 & 74.575 & 86.5 & 97.825 \\
\hline \multirow[t]{4}{*}{ Std.Dev. } & 2.7294688 & 0.2061553 & 0 & 5.6186446 & 5.5515764 & 0.697615 & 5.8323094 & 4.0542159 & 1.5370426 \\
\hline & & & & & & \multicolumn{4}{|c|}{ BRAIN } \\
\hline & & & & & & \multicolumn{4}{|c|}{ GRID SIZES(mm) } \\
\hline & & 3 & & & 5 & & \multicolumn{3}{|c|}{10} \\
\hline \multirow[t]{6}{*}{ (b) } & \multicolumn{3}{|l|}{$\begin{array}{c}\text { Gamma Function Test } \\
\text { Result (\%) }\end{array}$} & & & & & & \\
\hline & $2 \%, 2 \mathrm{~mm}$ & $3 \%, 3 \mathrm{~mm}$ & $5 \%, 5 \mathrm{~mm}$ & $2 \%, 2 \mathrm{~mm}$ & $3 \%, 3 \mathrm{~mm}$ & $5 \%, 5 \mathrm{~mm}$ & $2 \%, 2 \mathrm{~mm}$ & $3 \%, 3 \mathrm{~mm}$ & $5 \%, 5 \mathrm{~mm}$ \\
\hline & 96.3 & 99.5 & 100 & 88.9 & 96.3 & 99.5 & 71.7 & 84.8 & 95.5 \\
\hline & 93.9 & 100 & 100 & 88.2 & 90.1 & 100 & 82.6 & 89.4 & 99.9 \\
\hline & 99.5 & 100 & 100 & 86.2 & 96.6 & 99.5 & 75.2 & 90.1 & 98.5 \\
\hline & 94 & 100 & 100 & 71.2 & 90.1 & 94.1 & 65 & 88 & 96.3 \\
\hline Average & 95.925 & 99.875 & 100 & 83.625 & 93.275 & 98.275 & 73.625 & 88.075 & 97.55 \\
\hline \multirow[t]{6}{*}{ Std.Dev. } & 2.6285294 & 0.25 & 0 & 8.3619675 & 3.6682194 & 2.7932955 & 7.3286538 & 2.351418 & 2.0157712 \\
\hline & & & & & & \multicolumn{4}{|c|}{ PELVIS(Ca.Cx) } \\
\hline & & & & & & \multicolumn{4}{|c|}{ GRID SIZES(mm) } \\
\hline & & \multicolumn{2}{|c|}{3} & & 5 & & \multicolumn{3}{|c|}{10} \\
\hline & \multicolumn{3}{|l|}{$\begin{array}{c}\text { Gamma Function Test } \\
\text { Result }(\%)\end{array}$} & & & & & & \\
\hline & $2 \%, 2 \mathrm{~mm}$ & $3 \%, 3 \mathrm{~mm}$ & $5 \%, 5 \mathrm{~mm}$ & $2 \%, 2 \mathrm{~mm}$ & $3 \%, 3 \mathrm{~mm}$ & $5 \%, 5 \mathrm{~mm}$ & $2 \%, 2 \mathrm{~mm}$ & $3 \%, 3 \mathrm{~mm}$ & $5 \%, 5 \mathrm{~mm}$ \\
\hline \multirow[t]{4}{*}{ (c) } & 92.2 & 99.3 & 100 & 74.2 & 84.2 & 97.8 & 75.2 & 82.3 & 99.3 \\
\hline & 91.8 & 98.4 & 100 & 72.3 & 82.9 & 97.7 & 70.5 & 88.7 & 100 \\
\hline & 92 & 97.4 & 100 & 70 & 70 & 96.4 & 70.3 & 70 & 98.7 \\
\hline & 91 & 98 & 100 & 71.3 & 72 & 96 & 71.3 & 70.5 & 99.3 \\
\hline Average & 91.75 & 98.275 & 100 & 71.95 & 77.275 & 96.975 & 71.825 & 77.875 & 99.325 \\
\hline Std.Dev. & 0.5259911 & 0.7973916 & 0 & 1.7710637 & 7.3108937 & 0.9105859 & 2.291106 & 9.1863576 & 0.5315073 \\
\hline
\end{tabular}

Table 1: (a), (b) and (c) Summarizes the gamma passing criteria of head-and-neck, brain and pelvis (Ca.Cx.)

\begin{tabular}{|c|c|c|c|c|c|c|c|c|c|}
\hline & \multicolumn{6}{|c|}{ Averages of average of $2 \%, 2 \mathrm{~mm} 3 \%, 3 \mathrm{~mm}$ and $5 \%, 5 \mathrm{~mm}$ passing criteria } & \multirow{2}{*}{\multicolumn{2}{|c|}{10 mm(Grid Sizes) }} & \\
\hline & \multirow[b]{2}{*}{$2 \%, 2 \mathrm{~mm}$} & \multicolumn{2}{|c|}{$3 \mathrm{~mm}$ (Grid Sizes) } & \multirow[b]{2}{*}{$2 \%, 2 \mathrm{~mm}$} & \multicolumn{2}{|c|}{$5 \mathrm{~mm}$ (Grid Sizes) } & & & \\
\hline & & $3 \%, 3 \mathrm{~mm}$ & $5 \%, 5 \mathrm{~mm}$ & & $3 \%, 3 \mathrm{~mm}$ & $5 \%, 5 \mathrm{~mm}$ & $2 \%, 2 \mathrm{~mm}$ & $3 \%, 3 \mathrm{~mm}$ & $5 \%, 5 \mathrm{~mm}$ \\
\hline & 96.05 & 99.825 & 100 & 82.95 & 90.3 & 99.4 & 74.575 & 86.5 & 97.825 \\
\hline & 95.925 & 99.875 & 100 & 83.625 & 93.275 & 98.275 & 73.625 & 88.075 & 97.55 \\
\hline & 91.75 & 98.275 & 100 & 71.95 & 77.275 & 96.975 & 71.825 & 77.875 & 99.325 \\
\hline Average & 94.575 & 99.325 & 100 & 79.508333 & 86.95 & 98.216667 & 73.341667 & 84.15 & 98.233333 \\
\hline Std.Dev. & 2.44732 & 0.9096703 & 0 & 6.5544037 & 8.6661819 & 1.2135519 & 1.3967224 & 5.4910723 & 0.9553577 \\
\hline
\end{tabular}

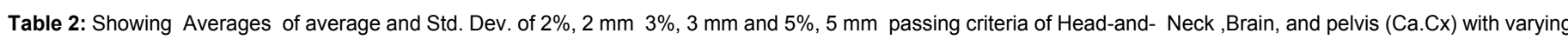
grid sizes.

$\mathrm{DD}$ and $5 \mathrm{~mm}$ DTA as the most suitable criteria for IMRT quality assurance. This gamma criterion of $5 \% \mathrm{DD}$ and $5 \mathrm{~mm}$ DTA favorably exceeds $95 \%$ in each case and grid sizes but it is not recommended for strict verification procedure in Intensity Modulated Radiotherapy (IMRT). The criteria of $2 \%$ DD and $2 \mathrm{~mm}$ DTA, and $3 \%$ DD and $3 \mathrm{~mm}$ DTA gamma values show below $90 \%$ for $5 \mathrm{~mm}$ and $10 \mathrm{~mm}$ grid sizes but exceeds $95 \%$ for the $3 \mathrm{~mm}$ grid sizes.

Hence $3 \mathrm{~mm}$ or less grid sizes should be routinely used in pretreatment verification procedure IMRT plans using 2D Array Detectors.

\section{Conflict of Interest}

The authors declare that they have no conflicts of interest. The authors alone are responsible for the content and writing of the paper.

\section{References}

1. Huang D, Xia P, Akazawa P, Akazawa C, Quivey JM, et al. (2003) Comparison of treatment plans using intensity-modulated radiotherapy and three-dimensional conformal radiotherapy for Para-nasal sinus carcinoma. Int J Radiat Oncol Biol Phys 56: 158-168.

2. Chao KS, Ozyigit G, Blanco Al, Thorstad WL, Deasy JO, et al. (2004) Intensity- 
Citation: Pathak P, Mishra PK, Birbiya N (2015) Dosimetric Study on Variations of Gamma Index (GI) in Pre-Treatment Verification Procedure in Intensity Modulated Radiotherapy (IMRT) Plans with Varying Grid Sizes Using 2D Array Detectors. J Cancer Sci Ther 7: 161-166. doi:10.4172/1948-5956.1000342

modulated radiation therapy for oropharyngeal carcinoma: Impact of the tumour volume. Int J Radiat Oncol Biol Phys 59: 43-50.

3. Miles EA, Clark CH, Urbano MT, Bidmead M, Dearnaley DP, et al. (2005) The impact of introducing intensity modulated radiotherapy into routine clinica practice. See comment in PubMed Commons below Radiother Oncol 77: 241 246.

4. Scott-Brown M, Miah A, Harrington K, Nutting C (2010) Evidence-based review: quality of life following head and neck intensity-modulated radiotherapy. See comment in PubMed Commons below Radiother Oncol 97: 249-257.

5. Nutting CM, Morden JP, Harrington KJ, Urbano TG, Bhide SA, et al. (2011) Parotid-sparing intensity modulated versus conventional radio-therapy in head and neck cancer (PARSPORT): a phase 3 multicentre randomized controlled trial. Lancet Oncol 12: 127-136.

6. Jursinic PA, Nelms BE (2003) A 2-D diode array and analysis software for verification of intensity modulated radiation therapy delivery. See comment in PubMed Commons below Med Phys 30: 870-879.

7. Nalbant N, Kesen ND, Hatice B (2014) Pre-Treatment Dose Verification of Imrt Using Gafchromic Ebt3 Film and 2d-Array. J Nucl Med Radiat Ther 5:182.

8. Elawady RA, Attalla EM, Elshemey WM, Shouman T, Alsayed AA (2014) Dose verification of intensity modulated radiotherapy in head and neck tumors. Int J Cancer Ther Oncol 2: 02037.
9. Mijheer B, Georg D (2008) Guidelines for the verification of IMRT. ESTRO BOOKLET NO. 9: Brussels, Belgium, 2008.

10. Ezzel G A, Galvin J M, Low D, Jatinder RP, Isaac R, et al. (2003) Guidance document on delivery, treatment planning, and clinical implementation of IMRT: report of the IMRT Subcommittee of the AAPM Radiation Therapy Committee. MedPhys 30: $2089-2115$.

11. Anjum MN, Parker W, Ruo R, Afzal M (2010) Evaluation criteria for film based intensity modulated radiation therapy quality assurance. See comment in PubMed Commons below Phys Med 26: 38-43.

12. Low DA, Moran JM, Dempsey JF, Dong L, Oldham M (2011) Dosimetry tools and techniques for IMRT. See comment in PubMed Commons below Med Phys 38: $1313-1338$

13. Agnew CE, Irvine DM, Hounsell AR, McGarry CK (2014) Improvement in clinical step and shoot intensity modulated radiation therapy delivery accuracy on an integrated linear accelerator control system. Pract Radiat Oncol 4: 43-49.

14. Escudé L, Linero D, Mollà M, Maralbell R (2006) Quality assurance for radiotherapy in prostate cancer: point dose measurements in intensity modulated fields with large dose gradients. Int JRadiat Oncol Biol Phys 66: S136-140.
Citation: Pathak P, Mishra PK, Birbiya N (2015) Dosimetric Study on Variations of Gamma Index (GI) in Pre-Treatment Verification Procedure in Intensity Modulated Radiotherapy (IMRT) Plans with Varying Grid Sizes Using 2D Array Detectors. J Cancer Sci Ther 7: 161-166. doi:10.4172/1948-5956.1000342 